\title{
AUTOMATED DETECTION AND ENHANCEMENT OF MICROCALCIFICATIONS IN MAMMOGRAMS USING NONLINEAR SUBBAND DECOMPOSITION
}

\author{
M. Nafi Gürcan ${ }^{1} \quad$ Yasemin Yardımci ${ }^{1} \quad$ A. Enis Çetin ${ }^{1} \quad$ Rashid Ansari ${ }^{2}$ \\ ${ }^{1}$ Bilkent University, \\ Dept. of Electrical and Electronics Engineering, \\ Bilkent, Ankara TR-06533, Turkey \\ E-mail: gurcan@ee.bilkent.edu.tr \\ ${ }^{2}$ Univ. of Illinois at Chicago \\ Department of Electrical Engineering \\ Chicago, Illinois, 60607-7053
}

\begin{abstract}
In this paper, computer-aided detection and enhancement of microcalcifications in mammogram images are considered. The mammogram image is first decomposed into subimages using a 'subband' decomposition filter bank which uses nonlinear filters. A suitably identified subimage is divided into overlapping square regions in which skewness and kurtosis as measures of the asymmetry and impulsiveness of the distribution are estimated. All regions with high positive skewness and kurtosis are marked as a regions of interest. Next, an outlier labeling method is used to find the locations of microcalcifications in these regions. An enhanced mammogram image is also obtained by emphasizing the microcalcification locations. Linear and nonlinear subband decomposition structures are compared in terms of their effectiveness in finding microcalcificated regions and their computational complexity. Simulation studies based on real mammogram images are presented.
\end{abstract}

\section{INTRODUCTION}

In this paper, computer-aided detection of microcalcifications and enhancement of digital mammogram images are considered. The presence of tiny calcium deposits in breast tissues are an early sign of breast cancer in women. Since microcalcifications can be as small as $0.1 \mathrm{~mm} \times 0.1 \mathrm{~mm}$ in size, they can be easily overlooked by an examining radiologist. Therefore, digitally enhanced mammogram images will help the diagnosis process.

Recently a variety of schemes for the computerized detection of microcalcifications, based on the wavelet transform, have been proposed [1]-[3]. In these schemes, the mammogram image is first passed through a subband decomposing filter bank. The subband images are weighted to enhance the microcalcification locations, and a new image is reconstructed from the weighted sub-images. In the detection step, global and local gray-level thresholds are applied to the reconstructed image to extract possible microcalcification locations. These locations are grouped to identify microcalcification clusters. In [1]-[3] the reconstructed image essentially corresponds to a bandpass filtered version of the original mammogram image.

In our method the mammogram image is first decomposed into subimages using a nonlinear subband decomposition structure and the microcalcification detection is carried out in the 'subband' domain. The nonlinear decomposition technique was recently proposed to compress images containing sharp edges [5]-[8]. The technique is also suitable for analyzing microcalcification locations which have also sharp edges.

Statistical procedures for detection of the microcalcifications are applicable after the decomposition stage. Since microcalcifications are small isolated regions in mammograms they produce outliers in the highband signal (the detail-image). The problem is then reduced to that of detecting outliers in the detail-image component, obviating the need for signal reconstruction. The detail-image is first divided into overlapping square regions in which skewness and kurtosis as measures of the asymmetry and impulsiveness of the distribution are estimated. A region with high positive skewness and kurtosis is marked as a region of interest. Then, the boxplot outlier detection method $[4,12]$ is used to find the locations of the microcalcifications in susceptible regions.

The performance of the nonlinear subband decomposition structure is also compared to that of regular filter banks employing linear filters.

\section{NONLINEAR IMAGE DECOMPOSITION}

In this section, the nonlinear image analysis structure is briefly reviewed, and it is shown that the choice of the nonlinear image decomposition structure, filters and their region of support should be determined according to the characteristics of microcalcifications.

The block diagram of a nonlinear signal decomposition structure is shown in Figure 1 [8]. In this structure $H$ and $G$ are nonlinear operators, and they produce the low-band signal, $y_{a}$, and the detail-signal, $y_{d}$ from the input signal $x$ as follows:

$$
\begin{aligned}
& y_{d}[n]=x_{2}(n)+H\left(\mathbf{x}_{1}[n]\right) \\
& y_{a}[n]=x_{1}(n)-G\left(\mathbf{y}_{d}[n]\right)
\end{aligned}
$$

where $x_{1}(n)=x(2 n-1), x_{2}(n)=x(2 n)$ and

$$
\begin{aligned}
& \mathbf{x}_{1}[n]=\left(x_{1}\left(n-N_{1}\right), \ldots, x_{1}(n), \ldots, x_{1}\left(n+N_{2}\right)\right), \\
& \mathbf{y}_{d}[n]=\left(y_{d}\left(n-N_{3}\right), \ldots, y_{d}(n), \ldots, y_{d}\left(n+N_{4}\right)\right),
\end{aligned}
$$

and $N_{1}, N_{2}, N_{3}, N_{4}$ are positive integers and they determine the support region of the nonlinear filters. The original signal $x$ can be perfectly reconstructed from the analysis signals $y_{a}$ and $y_{d}$, and the decomposition structure can be extended to two dimensions using either rectangular or quincunx subsampling methods [9]. 
The dimensions of microcalcifications vary in different mammogram images according to the scanning resolution. In the database we used, the scanning resolution is $100 \mu \mathrm{m} \times 100 \mu \mathrm{m}$ hence, the microcalcifications appear as impulses of size varying from 1 pixel $\times 1$ pixel to 10 pixel $\times 10$ pixel. Since 1 pixel $\times 1$ pixel microcalcifications can be removed by downsampling operation, the structure in Figure 1 cannot be used in mammogram image analysis with $100 \mu m \times 100 \mu m$ scanning resolution (It should be noted that the regular subband decomposition structures such as those in [1]- [3] can be used in image analysis, because the linear filters before the downsampling operation smear out the 1 pixel $\times 1$ pixel impulse and the information about it is available in all of the subsignals). If the scanning resolution is not sufficient then the downsampling operations should be eliminated and the subimages $y_{a}$ and $y_{d}$ are obtained as

$$
\begin{aligned}
& y_{d}[\underline{n}]=x[\underline{n}]-H(x[\underline{n}]), \\
& y_{a}[\underline{n}]=x[\underline{n}]+G\left(y_{d}[\underline{n}]\right)
\end{aligned}
$$

where the nonlinear filter $H$ is chosen as a median filter with a $21 \times 21$ support. Such a filter completely eliminates impulsive microcalcification regions while retaining the approximate base level. Therefore, the detail-signal $y_{d}$ contains mainly microcalcifications as shown in Figures 4-7 and the microcalcification locations can be determined using the statistical detection methods described in Section 3. Other nonlinear filters such as erosion and lower- $\alpha$ trimmed mean type filters can also be used for the $H$ filter. The nonlinear filter $G$, on the other hand, can be selected so that $y_{a}[\underline{n}]$ is an enhanced version of the original image $x[\underline{n}]$. This enhancement method will be introduced in Section 4 .

Nonlinear filters such as median type filters have been previously used in the detection of microcalcifications by Chang et.al. [11]. In [11] the effects of linear and nonlinear filters and their region of support in the detection and enhancement of microcalcifications are investigated through ROC studies. In [11] a median filter with a $9 \times 9$ support is found to be most effective on mammogram images with simulated microcalcifications. However, such a median filter cannot eliminate all of the microcalcifications in the mammogram images of Nijmejgen database ${ }^{1}$ because the sizes of the microcalcifications may exceed the median filter support region. The nonlinear filter support should be determined according to the image scanning rate and the sizes of microcalcifications.

The median filter with a support region containing $\mathrm{N}$ samples requires ' $2 \mathrm{~N}$ comparisons' to produce an output sample. On the other hand, a linear phase FIR filter requires N/4 multiplications. Furthermore, the microcalcification detection process can be carried out over quarter or half size images in regular subband decomposition structures. Therefore, the computational cost of a median filter based system is higher than an ordinary subband filterbank.

\section{STATISTICAL DETECTION METHOD}

Microcalcifications, tiny isolated regions in the mammogram image, manifest themselves as outliers in the detailimage. Hence, outlier detection techniques can be used to

\footnotetext{
${ }^{1}$ A set of mammogram images digitized by Nico Karssemeijer of University Hospital Nijmegen, The Netherlands.
}

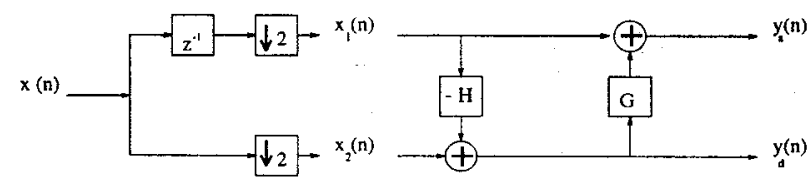

Figure 1. Nonlinear Subband Decomposition Structure

detect the microcalcifications [15]. In this paper, detection is carried out in two steps. First, the detail-image is divided into overlapping square regions. In these regions, skewness and kurtosis, measures of the asymmetry and impulsiveness of the distribution are estimated. If a region has high positive skewness and kurtosis then it is marked as a region of interest. In the second stage an outlier labeling method $[4,12]$ is used to find the locations of microcalcifications in these regions. This two step procedure significantly decreases the computational complexity because instead of searching the whole image for outliers only regions with high susceptibility are processed by the outlier labeling method.

\subsection{Skewness and Kurtosis Based Tests}

Skewness and kurtosis are higher order statistical parameters [13]. For a random variable $\mathbf{x}$, the skewness is defined as $[14]$

$$
\gamma_{3}=\frac{E\left[(\mathbf{x}-\mathrm{E}[\mathbf{x}])^{3}\right]}{\left(\mathrm{E}\left[(\mathbf{x}-\mathrm{E}[\mathbf{x}])^{2}\right]\right)^{3 / 2}}
$$

and is a measure of the symmetry of the distribution. An estimate of the skewness is given by:

$$
\hat{\gamma_{3}}=\frac{\sum_{i=1}^{N}\left(x_{i}-\hat{m}\right)^{3}}{(N-1) \hat{\sigma}^{3}}
$$

where $\hat{m}$ and $\hat{\sigma}$ are the estimates of the mean and standard deviation over $N$ observations $x_{i}(i=1, \ldots, N)$.

Similarly, for a random variable $\mathbf{x}$ the kurtosis is defined as

$$
\gamma_{4}=\frac{E\left[(x-E[x])^{4}\right]}{\left(E\left[(x-E[x])^{2}\right]\right)^{2}}-3
$$

and is a measure of the heaviness of the tails in a distribution. An estimate of the kurtosis is given by:

$$
\hat{\gamma}_{4}=\frac{\sum_{i=1}^{N}\left(x_{i}-\hat{m}\right)^{4}}{(N-1) \hat{\sigma}^{4}}-3
$$

where $\hat{m}$ and $\hat{\sigma}$ are defined as before. For the Gaussian distribution $\gamma_{3}$ and $\gamma_{4}$ are equal to zero.

If a region contains microcalcifications then due to the impulsive nature of microcalcifications the symmetry of the distribution of detail-image coefficients is destroyed as shown in Figure 2 (a) and (c). It is also evident that the tails of the distribution are heavier and hence the kurtosis assumes a high value. Therefore a statistical test based on skewness and kurtosis is effective in finding regions with asymmetrical and heavier tailed distributions. The detection problem is posed as an hypothesis testing problem in which the null hypothesis, $H_{o}$, corresponds to the case of no microcalcifications against the alternative $H_{1}$ :

- $H_{o}$ : Microcalcifications are not present in the region

- $H_{1}$ : Microcalcifications are present in the region. 

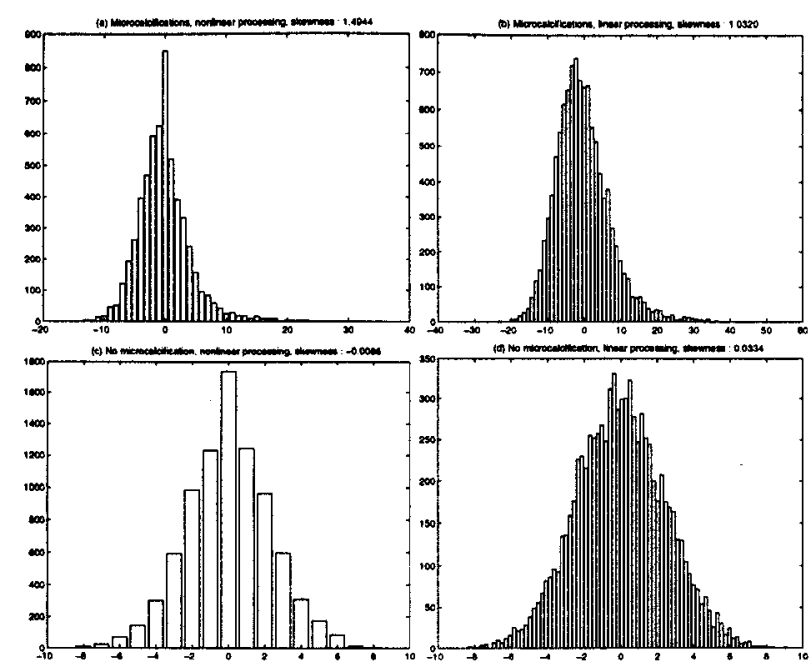

Figure 2. Sample value distributions in regions with nonlinear subband decomposition (left column) and with linear subband decomposition (right column)

This hypothesis testing problem is reduced to the following decision rule $\Gamma$ based on skewness and kurtosis

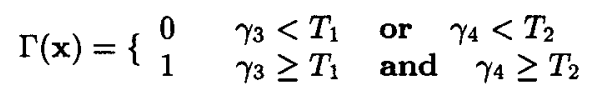

where $T_{1}$ and $T_{2}$ are experimentally determined thresholds.

Once the regions containing microcalcifications are determined by the above test, the locations of the microcalcifications are estimated by the outlier labeling method described in the next subsection.

In [15] a linear subband decomposition filter bank is used instead of the nonlinear subband decomposition structure of Section 2. In Figure 2 (b) and (d) detail-image histograms of two regions obtained with a linear structure are shown. The region containing microcalcifications has high skewness and kurtosis values, too. Basically, the linear and nonlinear structures both reveal the same information in terms of their higher order statistical parameters. The computational complexity of the nonlinear subband decomposition structure is high compared to that of the linear structure.

\subsection{Boxplot Outlier Labeling Method}

In this work, we used the so-called boxplot outlier labeling method [12]. In this method the data $\mathbf{x}$ is first rank ordered, $\mathbf{x}=\left\{x_{(1)}, x_{(2)}, \ldots \ldots, x_{(n)}\right\}$. Next, the median, the lower quartile, $Q_{1}$ and the upper quartile $Q_{3}$ values are determined [15]. The interquartile range $R_{F}$ is defined to be $Q_{3}-Q_{1}$. The boxplot method determines the outliers to be the part of data which is outside the region $\left(Q_{1}-k R_{F}, Q_{3}+k R_{F}\right)$. The parameter $k$ is usually taken to be 1.5 or 3.0 . Figure 3 illustrates the boxplot outlier labeling method.

\section{MAMMOGRAM IMAGE ENHANCEMENT}

It is desired that the microcalcifications be readily noticeable in an enhanced version of the original mammogram image. Expression (4) shows that the image $y_{a}[\underline{n}]$ is obtained

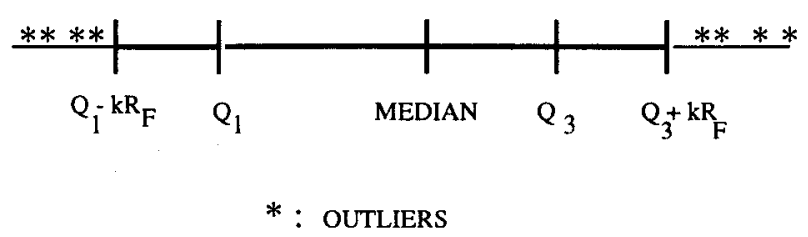

Figure 3. Boxplot outlier labeling method definitions

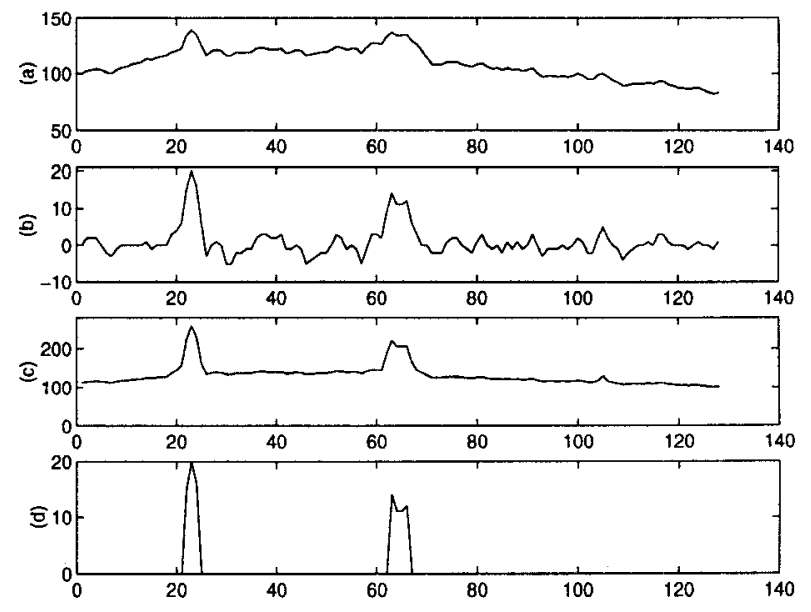

Figure 4. (a) A horizontal line of the mammogram image, (b) detail-image $y_{d}$, (c) microcalcifications are enhanced (d) output of the outlier detection method.

by superimposing the original with a nonlinear filtered version of the detail-image $G\left(y_{d}[\underline{n}]\right)$. The image $y_{a}[\underline{n}]$ can be considered as an enhanced image for an appropriate selection of the nonlinear function $G$. A natural choice for $G$ would be based on the outlier detection scheme. In this approach, the microcalcification regions in $y_{d}[\underline{n}]$ are detected and reinforced. Another approach is predicated on amplifying the pixel values of the original at the microcalcification locations.

Figure 4 illustrates the steps of outlier detection and image enhancement stages on a horizontal line of a mammogram image. In particular, Figure 4 (a) depicts the original line which is known to contain two microcalcifications. The difference between the original signal and its median filtered version is shown in Figure 4 (b). This difference plot corresponds to the detail-signal, $y_{d}$ of the nonlinear decomposition structure. The microcalcifications are en-

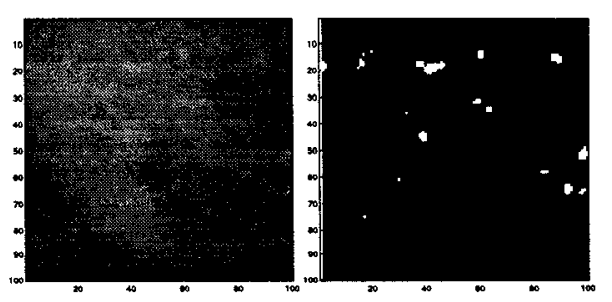

Figure 5. (a) A region of a mammogram image containing microcalcifications, (b) output of the detection scheme 


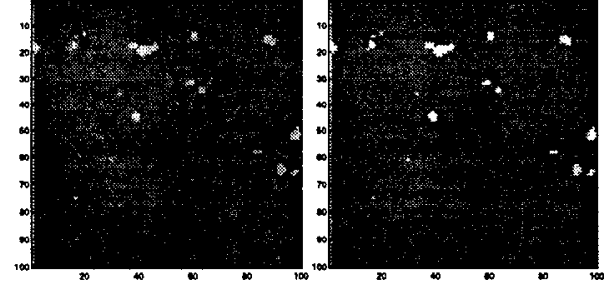

Figure 6. Enhanced Images (a) Microcalcifications are superimposed on the mammogram image, (b) Mammogram image is magnified at the locations of microcalcifications

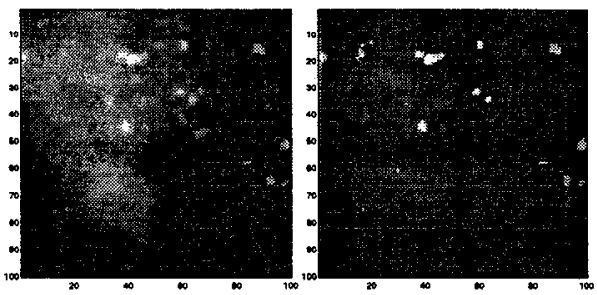

Figure 7. Enhanced and contrast stretched images (a) and (b) as defined in Figure 6.

hanced in Figure 4 (c). Figure 4 (d) illustrates the output of the outlier detection scheme. In the detection, only the detail-signal, $y_{d}$, is used. Figure 5 (a) shows a part of a mammogram image with a cluster of microcalcifications. In Figure 5 (b), the output of the microcalcification detection scheme is shown. Figure 6 illustrates the enhanced images for two approaches: superimposition and magnification.

In case enhancement of the tissue around the microcalcification regions is also desired, the final image can be displayed after scaling so that the full dynamic range of the display device is employed. Figure 7 shows the results of such a contrast stretching algorithm.

In these figures the microcalcifications are successfully detected and mammogram images are enhanced. Additionally, the processing is simple, and does not require a full decomposition and reconstruction.

\section{CONCLUSION}

In this paper, automatic detection of microcalcifications in mammogram images is considered. The mammogram image is first processed by a nonlinear subband decomposition filter bank. Microcalcifications, tiny, isolated regions, produce outliers in the detail-image. Next, the the detailimage is divided into overlapping square regions in which skewness and kurtosis are estimated. These higher order statistical parameters are measures of the asymmetry and impulsiveness of the distribution. Therefore a region with high positive skewness and kurtosis is marked as a region of interest. Finally, an outlier labeling method is used to find the locations of microcalcifications in these regions.

Subband decomposition structures both using linear and nonlinear filters are also examined. Both structures are successful in identifying regions with microcalcifications. The computational complexity of the linear subband decomposition structure is low compared to the nonlinear subband decomposition structure.

\section{REFERENCES}

[1] H. Yoshida, K. Doi, R. M. Nishikawa, "Automated detection of clustered microcalcifications in digital mammograms using wavelet transform techniques," Proc. SPIE, 2167:868-886, 1994

[2] H. Yoshida, W. Zhang, W. Cai, K. Doi, R. M. Nishikawa and M. L. Giger, "Optimizing Wavelet Transform Based On Supervised Learning For Detection of Microcalcifications in Digital Mammograms," Proceedings of IEEE International Conference on Image Processing, pp. 152-155 1995.

[3] R. N. Strickland, H. I. Hahn, "Wavelet Transform Matched Filters For the Detection and Classification of Microcalcifications in Mammography," Proceedings of IEEE International Conference on Image Processing, pp. 422-425, 1995.

[4] Y. Barnett, T. Lewis, Outliers in Statistical Data, 3rd Ed. New York: John Wiley \& Sons, 1994.

[5] O. Egger, M. Kunt, "Embedded Zerotree Based Lossless Image Coding," IEEE ICIP'95, vol. II pp. 616619., June 1995.

[6] D. E. F. Florencio, R. Schafer "Perfect Reconstructing Nonlinear Filter Banks," in ICASSP'96, 1996.

[7] R. L. de Queiroz, D. A. F. Florencio, "A Pyramidal Coding Using a Nonlinear Filter Bank," in ICASSP'96, 1996.

[8] F. J. Hampson, J. C. Pesquet, "A Nonlinear Subband Decomposition with Perfect Reconstruction," in ICASSP'96, 1996.

[9] R. Ansari, A. E. Cetin, S. H. Lee, "Subband Coding of Images Using Nonrectangular Filter Banks," in SPIE Vol.974, Applications of Digital Image Processing XI, pp. 315-322, 1988.

[10] I. Pitas, A. N. Venetsanopoulos, Nonlinear Digital Filters - Principles and Applications, Massachusetts: Kluwer Academic Publishers, 1990.

[11] H. Chang, K. Doi, S. Galhotra, C. J. Vyborny, H. MacMahon, P. M. Jokich, "Image feature analysis and computer-aided diagnosis in digital radiography: I. Automated detection of microcalcifications in mammography," Medical Physics, Vol. 14, No. 4, Jul/Aug 1987.

[12] B. Iglewicz, D. C. Hoaglin, How to Detect and Handle Outliers, ASQC basic references in quality control; v.16, 1993.

[13] C. L. Nikias, A. Petropulu, Higher Order Statistical Analysis, Prentice Hall, 1994.

[14] P. J. Bickel, K. A. Doksum, Mathematical Statistics, Holden-Day California : 1977.

[15] M. N. Gürcan, Y. Yardımcı, A. E. Çetin, Rashid Ansari, "Detection of microcalcifications in mammograms using nonlinear subband decomposition and outlier labeling," in Proceedings of SPIE Visual Communications and Image Processing Conference, 8-14 February, 1997, San Jose, CA. 